\title{
La inclusión educativa desde la voz de madres de estudiantes con Trastornos del Espectro Autista en una muestra chilena
}

\author{
Inclusive education from the point of view \\ of the mothers of students with Autism \\ Spectrum Disorder in a Chilean sample
}

\section{Resumen}

El objetivo del estudio es mostrar la perspectiva de madres chilenas sobre la inclusión educativa de sus hijos escolarizados en centros de educación primaria, quienes presentan un Trastorno del Espectro Autista. Se recogen evidencias, desde la voz de las familias, que pueden servir como punto de partida para reflexionar sobre cómo avanzar hacia una educación más inclusiva y cómo contribuir a mejorar los apoyos que se brindan a estudiantes con discapacidad. El estudio se enmarca en un paradigma cualitativo de investigación, utilizando entrevistas no estructuradas para acceder a la información. Se ha contado con la participación de ocho madres chilenas de la Región de Valparaíso. Los resultados muestran que la valoración de la escolarización está estrechamente relacionada con el bienestar emocional, ámbito al que se le otorga mayor relevancia que al de los resultados académicos. Se destaca la importancia de los apoyos adaptados a las necesidades del sistema familiar.

\section{Palabras clave}

Inclusión educativa, discapacidad y familia, voces de las familias.

\begin{abstract}
The purpose of the study is to show the perspective of Chilean mothers on the educational inclusion of children who have Autism Spectrum Disorder enrolled in primary schools. Evidence was collected by listening to the voices of families, which can serve as a starting point to reflect on how to move towards a more inclusive education and how to help improve the supports that are being provided to students with disabilities. The study was based on a qualitative research paradigm, using unstructured interviews to access the information. It counted with the participation of eight Chilean mothers from the Region of Valparaíso. The results show that the value of schooling is closely related to the emotional well-being, which is given greater significance than the academic results. The importance of supports adapted to the needs of the family system is emphasized.
\end{abstract}

\section{Keywords}

Educational inclusion, disability and family, voices of families.

\section{Marcela Villegas Otárola \\ villegasmp@gmail.com}

Doctoranda Universidad Autónoma de Madrid

\section{Cecilia Simón Rueda}

cecilia.simon@uam.es

Universidad Autónoma de Madrid

\section{Gerardo Echeita \\ Sarrionandia}

gerardo.echeita@uam.es

Universidad Autónoma de Madrid
Para citar:

Villegas Otárola, M.; Simón Rueda, C. y Echeita Sarrionandia, G. (2OI4):

"La inclusión educativa desde la voz de madres de estudiantes con Trastornos del Espectro Autista en una muestra chilena", Revista Española de Discapacidad, 2 (2): 63-82.

<http://dx.doi.org/IO.5569/23405 I04.02.02.04>

Fecha de recepción: I 5-O2-20I4 Fecha de aceptación: O5-I I-20I4 


\section{Introducción}

Para los propósitos del estudio entendemos la inclusión educativa como el proceso de cambio sistemático que intenta promover la participación del alumnado en la cultura, la comunidad y el currículo, a través de procesos de mejora e innovación educativa dirigidos a eliminar las barreras que limitan su presencia, aprendizaje y participación en los centros donde son escolarizados, con especial atención a aquellos más vulnerables de ser excluidos (Ainscow, Booth y Dyson, 2006).

Ahora bien, cabe preguntarse qué condiciones son fundamentales para que las escuelas puedan avanzar hacia una comunidad escolar más inclusiva, que asegure la equiparación de oportunidades a sus estudiantes, que sea respetuosa con las diferencias individuales y comprensiva con el alumnado más vulnerable de ser excluido.

Al respecto, Ainscow et al. (2OI2) señalan que, para enfrentar el desafío de avanzar hacia una educación más inclusiva desde la propia realidad educativa, resulta crítico usar evidencias que promuevan la reflexión y los procesos de mejora e innovación dentro de la escuela. Es a la hora de recoger esas evidencias cuando el escuchar las voces de los involucrados, entre ellas las de los alumnos, familias y profesionales, cobra gran relevancia y adquiere un protagonismo que pocas veces ha tenido en las iniciativas de mejora educativa. En este mismo sentido podemos señalar que la participación de la familia en los procesos educativos de estudiantes con discapacidad en la escuela regular viene a ser, en palabras de Ainscow, Booth y Dyson (2006), una de las palancas de cambio para lograr un sistema educativo más comprensivo con la diversidad.

La relevancia de abordar el estudio de la inclusión educativa de estudiantes con discapacidad desde la perspectiva de las familias se fundamenta, además, por el importante rol que han de cumplir en los procesos educativos en los que participan junto a sus hijos e hijas, como también en el papel decisivo que han tenido a la hora de promover e impulsar la inclusión educativa (Moliner, 2008). Del mismo modo, conocer la perspectiva de las familias sobre la inclusión educativa resulta fundamental, también, por la importante información que aportan sobre el estudiante que se incluye, siendo un recurso natural de apoyo en los diferentes contextos de desarrollo (Turnbull, 2003). Adicionalmente, las familias pueden proporcionar información y hacer valoraciones respecto de las prácticas y políticas inclusivas, lo que puede ser de gran valor a la hora de evaluar sistemáticamente los programas de inclusión (Salend y Garrick, 2002). Su participación resulta fundamental, finalmente, porque la inclusión comienza en el seno de la propia familia, por lo que el sistema educativo debe reconocer su derecho a participar en las decisiones que involucran a sus hijos e hijas, ya que son centrales a la hora de proteger $y$ defender sus derechos (UNESCO, 2004).

En relación con la participación de la familia en la planificación de los apoyos para estudiantes con discapacidad que acceden a la educación regular, diversos estudios en relación a la prestación de servicios en el ámbito de la discapacidad insisten en la relevancia de construir relaciones colaborativas con las familias y considerar su perspectiva a la hora de planificarlos (Dobbins y Abbott, 20го; Giné, 2004; Parsons, Lewwis y Ellins, 2009; Shippers y Van Boheemen, 2009; Turnbull, 2003; Turnbull, Turnbull y Kyzar, 2009). Ellas son una fuente fundamental de información sobre las necesidades de sus hijos, algo esencial, como ya hemos señalado, para que los procesos educativos inclusivos sean efectivos (Salend y Garrick, 2002; Verdugo y Rodríguez, 20I2).

Estas relaciones colaborativas y de cooperación entre familias y profesionales, necesariamente, han de construirse sobre la comunicación, el respeto, el compromiso y la confianza mutua, lo que solo es posible si favorecemos su participación y consideramos sus opiniones y perspectivas a la hora de llevar a cabo los procesos educativos en los que participan sus 
hijos, convirtiéndose de esta forma en una fuerza catalizadora de la inclusión (Turnbull, Turnbull y Kyzar, 2009).

En relación con los estudios sobre inclusión educativa desde la perspectiva de las familias, si bien contamos con trabajos en esta dirección (ver, por ejemplo, Frederickson et al., 2004; González del Yerro et al., 2013; Kluth et al., 2007; O’Connor, 2007; Rogers, 2007; Verdugo y Rodríguez, 20I2; Yssel et al., 2007), las iniciativas llevadas a cabo desde su voz aún son escasas, principalmente en el contexto hispanoamericano. Los estudios llevados a cabo muestran que, a pesar de que se reconoce el valor de la escolarización en escuelas regulares, muchas de las familias con hijos o hijas con discapacidad consideran que hay múltiples situaciones por mejorar dentro de la educación regular, que originan muchas limitaciones y dificultades en los procesos educativos.

Simón y Echeita (2010) recogen algunas de las principales preocupaciones de las familias, a partir de la revisión de los trabajos realizados con familias de estudiantes con discapacidad que han accedido a la educación regular, por ejemplo, carencias en la experiencia de implementación de la inclusión, poca aceptación de alumnos con discapacidad por parte de los centros educativos y falta de personal cualificado para concretarla. Asimismo, dan cuenta de limitaciones en la atención individualizada requerida por los alumnos, en el intercambio de información entre padres y profesionales, lo cual se traduce en una percepción de falta de comunicación y dificultades en la coordinación entre ambos, así como una carencia de recursos para atender a la diversidad existente en el aula.

En el caso específico de los trabajos sobre inclusión educativa desde la perspectiva de familias de estudiantes con Trastornos del Espectro Autista (en adelante TEA) aún es necesario contar con más evidencias (Jackson, Renwick y Fudge, 2008; Parsons, Lewis y Ellins, 2009). No obstante, estos estudios nos permiten señalar que, si bien la inclusión educativa se vive como un desafío por parte de la mayoría de las familias con hijos con necesidades de apoyo, y que su éxito o fracaso tiene una alta incidencia en el bienestar emocional del grupo familiar, la escolarización de hijos con necesidades de apoyo causadas por un TEA supone, para ellas, una situación especialmente compleja y preocupante (Parsons, Lewis y Ellins, 2009). Estas familias perciben que es más difícil acceder a servicios educativos de calidad para sus hijos en comparación con la situación de familias con hijos con otra discapacidad (Parsons, Lewis y Ellins, 2009).

Respecto al contexto en el que se lleva a cabo nuestro estudio, el contexto educativo chileno, se puede señalar que, en relación con las oportunidades educativas existentes en Chile para los estudiantes con discapacidad, hasta la fecha, se siguen contemplando dos alternativas (MINEDUC, 2005), los establecimientos de Educación Regular con Proyectos de Integración Escolar (PIE) ${ }^{\mathrm{I}}$ y las Escuelas Especiales.

En el año 20ıo, de acuerdo con el Sistema de Información General de Estudiantes del MINEDUC, el 3,9\% de la matrícula nacional asiste a escuelas especiales para estudiantes con algún tipo de discapacidad y solo un $0,4 \%$ de la matrícula total asiste a un PIE. Esta situación es realmente crítica cuando comprendemos que, en el ámbito de la discapacidad, la educación formal cobra un carácter estratégico, siendo la puerta de entrada para la inclusión social (Echeita y Simón, 2007). Esta situación acontece con independencia de que el Estado de Chile se ha sumado a las convenciones de derechos humanos más relevantes en la materia, como es el caso de la Declaración y Marco de Acción de Salamanca para las Necesidades Educativas Especiales (UNESCO, I994), la Convención de los Derechos de las Personas con Discapacidad (ONU, 2006) y la Convención de Naciones Unidas sobre los Derechos del Niño (ONU, I989).

A la luz de lo anteriormente expuesto, el objetivo general de nuestro estudio ha sido

\footnotetext{
I. Cabe señalar que en Chile aún se habla de "integración escolar". Actualmente este concepto se utiliza como sinónimo de "inclusión educativa", a pesar de las diferencias conceptuales y prácticas que existen entre ambos conceptos.
} 
conocer la perspectiva de un grupo de madres de hijos o hijas con TEA sobre la inclusión educativa y sus procesos de escolarización en la escuela ordinaria, con el fin de recoger evidencias, desde las voces de las propias familias, que sirvan como punto de partida para la reflexión en torno a cómo construir comunidades educativas cada vez más inclusivas y contribuir a mejorar los apoyos que se brindan a los estudiantes con discapacidad que acceden a la educación regular y a sus familias.

En la consecución de ese objetivo se formularon cinco preguntas de investigación, que orientaron el análisis y la organización de los resultados:

a. ¿Cuál es la perspectiva de algunas madres chilenas de estudiantes con TEA sobre el proceso de inclusión educativa?

b. ¿Qué valoración hacen las madres respecto de los procesos de escolarización vividos con sus hijos?

c. ¿Cuál es la perspectiva sobre los apoyos recibidos por sus hijos y sus familias durante los procesos de escolarización en los que participan?

d. ¿Qué barreras y facilitadores identifican en los procesos de escolarización vividos?

e. ¿Qué propuestas realizan para avanzar hacia una educación más inclusiva?

\section{Método}

El estudio se enmarca en un paradigma cualitativo de investigación. Se trata de un estudio, descriptivo fenomenológico (Mertens, 20Io), que utiliza una entrevista no estructurada con una muestra de madres chilenas de estudiantes con TEA que han accedido a la educación regular.

\subsection{Participantes}

Participaron en este estudio ocho madres chilenas de estudiantes que presentan una discapacidad asociada a un TEA, cuyas edades fluctúan entre los 8 y I 2 años de edad, quienes asisten a seis escuelas primarias² de la Región de Valparaíso: una escuela pública, tres escuelas concertadas y dos escuelas privadas. El grupo de participantes corresponde a la totalidad de madres de estudiantes con TEA que asisten a las seis escuelas consideradas para el estudio. Los hijos de las participantes tienen, al menos, un año lectivo de escolaridad en sus respectivas escuelas.

Respecto al diagnóstico de TEA, estos han sido emitidos previamente por profesionales especialistas registrados y certificados por el MINEDUC, a partir de los criterios diagnósticos del Diagnostic and Statistical Manual of Mental Disorders, DSM IV.

Resulta importante destacar que las ocho participantes del estudio constituyen un grupo excepcional en relación con la población de estudiantes con TEA de la Región de Valparaíso, en consideración a que la mayoría de ellos se encuentran escolarizados en escuelas especiales para estudiantes con TEA o Discapacidad Intelectual.

Inicialmente en este estudio se invitó a participar tanto a padres como a madres de estudiantes con TEA. Sin embargo, únicamente respondieron a la solicitud las madres.

Respecto a la confidencialidad y el anonimato, este se garantizó a través de un consentimiento informado en el que se aseguró la confidencialidad de la información durante todo el proceso, el anonimato en la transcripción, tratamiento de los datos y en la difusión de los resultados.
2. El sistema educativo chileno contempla emplazamientos de tres tipos de dependencia; los llamados públicos, en los cuales existe un sistema de municipalización en el cual cada municipio administra las escuelas de su localidad; los particulares subvencionados (concertados) cuya de dependencia es privada, pero cuenta con subvención estatal; los privados sin subvención estatal. 


\subsection{Instrumentos}

Se llevó a cabo una entrevista no estructurada, complementada con un protocolo de registro de información social y demográfica de la familia.

Este protocolo permitió recoger información descriptiva sobre la familia y sobre el hijo con TEA. Respecto al diseño de la entrevista, se establecieron previamente cinco dimensiones de análisis teóricas, concretadas en catorce categorías específicas, que permitieron construir una guía de entrevista para los participantes del estudio.

La Tabla I da cuenta de las dimensiones, categorías específicas e indicadores utilizados para la formulación de la guía de entrevista.

\section{Tabla 1. Estructura utilizada para la elaboración de la guía de entrevista}

\begin{tabular}{|c|c|c|}
\hline $\begin{array}{l}\text { Dimensiones de } \\
\text { análisis }\end{array}$ & Categorías específicas & Indicadores \\
\hline \multirow{2}{*}{$\begin{array}{l}\text { Conocimientos } \\
\text { sobre Inclusión } \\
\text { Educativa (IE) }\end{array}$} & Elementos definitorios de la IE & $\begin{array}{l}\text { - Conceptualización de la IE } \\
\text { - Aspectos positivos y negativos de la IE } \\
\text { - Conceptualización de calidad educativa }\end{array}$ \\
\hline & $\begin{array}{l}\text { Normativa que regula el acceso de } \\
\text { estudiantes con discapacidad a la } \\
\text { escuela ordinaria }\end{array}$ & $\begin{array}{l}\text { - Conocimiento de la normativa } \\
\text { - Valoración de la normativa }\end{array}$ \\
\hline \multirow{6}{*}{$\begin{array}{l}\text { Vivencias de } \\
\text { escolarización }\end{array}$} & Trayectoria escolar & $\begin{array}{l}\text { - Educación Especial o Educación Regular } \\
\text { - Ingreso a la Educación Regular } \\
\text { - } \text { Modalidad de IE } \\
\text { - Bienestar del estudiante en su comunidad } \\
\text { escolar }\end{array}$ \\
\hline & Presencia & $\begin{array}{l}\text { - Asistencia a la jornada escolar } \\
\text { - Asistencia a las actividades extracurriculares } \\
\text { de la escuela }\end{array}$ \\
\hline & Aprendizaje & $\begin{array}{l}\text { - } \text { Acceso al aprendizaje } \\
\text { - Responsabilidad ante el aprendizaje } \\
\text { - Satisfacción con resultados de aprendizaje } \\
\text { - Individualización del currículum en relación } \\
\text { con las necesidades de apoyo }\end{array}$ \\
\hline & Participación & $\begin{array}{l}\text { - Aceptación del estudiante en la escuela } \\
\text { - Relación con los compañeros } \\
\text { - Relación con los profesores } \\
\text { - Ausencia de situaciones de maltrato o abuso } \\
\text { de poder }\end{array}$ \\
\hline & Familia en la escuela & $\begin{array}{l}\text { - Participación de la familia en la escuela } \\
\text { - Participación de la familia en el proceso } \\
\text { - Relación de la familia con la escuela } \\
\text { - Relación de la familia con otras familias }\end{array}$ \\
\hline & $\begin{array}{l}\text { Gestión y cultura } \\
\text { escolar }\end{array}$ & $\begin{array}{l}\text { - Actitud del docente frente al estudiante } \\
\text { - Actitud de la escuela frente a la diversidad } \\
\text { - Organización institucional } \\
\text { - Organización del aula }\end{array}$ \\
\hline \multirow{2}{*}{ Apoyos } & $\begin{array}{l}\text { Apoyos requeridos por el } \\
\text { estudiante }\end{array}$ & $\begin{array}{l}\text { - Necesidades de apoyo del estudiante } \\
\text { - Apoyos brindados al estudiante } \\
\text { - Valoración de apoyos brindados al estudiante }\end{array}$ \\
\hline & Apoyos requeridos por la familia & $\begin{array}{l}\text { - Necesidades de apoyo de la familia. } \\
\text { - Apoyos brindados a familia, } \\
\text { - Valoración de apoyos brindados a la familia. }\end{array}$ \\
\hline
\end{tabular}




\begin{tabular}{|l|l|l|}
\hline $\begin{array}{l}\text { Dimensiones de } \\
\text { análisis }\end{array}$ & Categorías específicas & Indicadores \\
\hline \multirow{2}{*}{$\begin{array}{l}\text { Impacto de la } \\
\text { escolarización sobre } \\
\text { la familia }\end{array}$} & Respecto de la madre & $\begin{array}{l}\bullet \text { Bienestar emocional de la madre } \\
\text { Expectativas }\end{array}$ \\
\cline { 2 - 3 } & Respecto del grupo familiar & $\begin{array}{l}\bullet \text { Interacción familiar } \\
\text { Recursos económicos }\end{array}$ \\
\hline $\begin{array}{l}\text { Propuestas de } \\
\text { mejoras }\end{array}$ & A la escuela & $\bullet$ Sugerencias para la escuela \\
\cline { 2 - 3 } & Al sistema educativo & $\bullet$ Sugerencias para el sistema educativo \\
\hline
\end{tabular}

Cabe señalar que se desarrolló una entrevista preliminar con un grupo piloto compuesto por dos participantes, con el fin de evaluar la pertinencia de las dimensiones de análisis, las categorías específicas, la comprensión de las preguntas, el tiempo de duración de la entrevista $\mathrm{y}$, en definitiva, la correspondiente guía de entrevista.

\subsection{Procedimiento}

Cada participante completó el protocolo de registro de información social y demográfica de la familia y, posteriormente, se llevaron a cabo las entrevistas. Éstas se efectuaron de manera individual, en sesiones únicas de 90 a I 20 minutos, con un descanso a los 60 minutos, y fueron grabadas con la autorización de las madres, recogida con la firma del consentimiento informado. Todas las entrevistas fueron realizadas por la misma investigadora. Las entrevistas grabadas fueron transcritas y para facilitar su análisis, para ello se utilizó el programa informático Atlas. ti 5.o. El proceso seguido se realizó conforme a las recomendaciones de Braun y Clarke (2006), al respecto. En un primer momento se desarrolló una lectura reflexiva del registro y se identificaron las ideas fundamentales en cada respuesta, las que constituyen los códigos emergentes preliminares (CEP) del estudio. Posteriormente se procedió a codificar los textos y los códigos emergentes preliminares pasaron a ser nuestros códigos emergentes definitivos (CED). A continuación se revisó la asignación de códigos contrastando su asignación con la definición elaborada para cada uno de ellos. Por último, se confirman los códigos emergentes definitivos usados para el análisis de las entrevistas. Cabe señalar que en esta codificación definitiva se llevó a cabo un proceso de fiabilidad interjueces, utilizando para ello el $20 \%$ del total de las citas analizadas. El porcentaje de acuerdo con cada juez fue; $95 \%$ de acuerdo con el juez I; $96 \%$ con el juez 2; $93 \%$ con el juez 3 . Se puede señalar un promedio de acuerdo de $95 \%$.

\section{Resultados}

El proceso de análisis llevado a cabo para acceder a la perspectiva de las madres sobre la inclusión educativa y la escolarización de sus hijos ha dado como resultado 6I I citas textuales y 39 códigos emergentes definitivos .

En la Tabla 2 se presenta un resumen de los códigos emergentes definitivos, incluidos dentro de cada dimensión y categoría, así como el porcentaje de aparición de citas y ejemplos de citas representativas. Respecto de los porcentajes que acompañan a cada categoría, es necesario explicitar que estos se obtienen en función de su aparición dentro del Io०\% de citas utilizadas.

En cuanto a la organización de los resultados, estos son presentados en función de las cinco dimensiones de análisis consideradas para este estudio. Estas dimensiones permiten dar respuesta a nuestras preguntas de investigación. 
Tabla 2. Resumen de códigos emergentes definitivos (CED) y frecuencias de citas por categorías

\begin{tabular}{|c|c|c|c|c|}
\hline Dimensiones & Categorías & $\%$ & Códigos emergentes definitivos & Citas \\
\hline \multirow{2}{*}{$\begin{array}{l}\text { 1. Conocimiento } \\
\text { sobre Inclusión } \\
\text { Educativa (IE) }\end{array}$} & $\begin{array}{l}\text { 1.1. Elementos } \\
\text { definitorios de } \\
\text { la IE }\end{array}$ & 5,3 & $\begin{array}{l}\text { - IE es un derecho } \\
\text { - IE contempla aprendizaje y desarrollo } \\
\text { - IE contempla presencia } \\
\text { - IE contempla bienestar y participación } \\
\text { - IE contempla provisión de apoyos }\end{array}$ & $\begin{array}{l}\text { "que esté inserto pero que también sea } \\
\text { parte, no que esté ahí sentado haciendo } \\
\text { nada, que esté dentro del aula y participe, } \\
\text { ahora, obviamente la inclusión educativa va } \\
\text { a durar hasta que T se sienta cómodo en ese } \\
\text { sistema" }\end{array}$ \\
\hline & $\begin{array}{l}\text { 1.2. Normativa } \\
\text { que regula } \\
\text { el acceso de } \\
\text { estudiantes con } \\
\text { discapacidad a la } \\
\text { escuela ordinaria }\end{array}$ & 2,0 & $\begin{array}{l}\text { - Conocimiento de la normativa } \\
\text { - Valoración de la normativa }\end{array}$ & $\begin{array}{l}\text { "en el papel es medianamente buena y en la } \\
\text { práctica es muy mala, la normativa es muy } \\
\text { segregadora porque habla de que la inclusión } \\
\text { sí existe pero los que tienen la obligación, } \\
\text { entre comillas, porque hasta ahora es } \\
\text { opción de los colegios el tener proyectos de } \\
\text { integración, ni siquiera de inclusión" }\end{array}$ \\
\hline \multirow{5}{*}{$\begin{array}{l}\text { 2. Vivencias de } \\
\text { escolarización }\end{array}$} & $\begin{array}{l}\text { 2.1. Trayectoria } \\
\text { escolar }\end{array}$ & 33,0 & $\begin{array}{l}\text { - Acceso a la educación regular } \\
\text { - Educación Especial v/s } \\
\text { - Educación Regular } \\
\text { - Calidad educativa } \\
\text { - Cambios de Escuela } \\
\text { - Bienestar del estudiante } \\
\text { - Relación familia-escuela } \\
\text { - Relación con otras familias } \\
\text { - Consideración para la toma de decisiones } \\
\text { - Participación de la familia en la escuela } \\
\text { - Valoración de la IE }\end{array}$ & $\begin{array}{l}\text { "yo empecé a buscar el colegio pero me } \\
\text { costó tanto, recorri tantos colegios y me } \\
\text { decían de todo, en algunos casos no, no } \\
\text { recibimos niños así o que sus características } \\
\text { les complicaban, que había que hablarlo con } \\
\text { la tía primero, algunas tías me decían que no } \\
\text { se sentían capacitadas para un niño de este } \\
\text { tipo, de esa clase" }\end{array}$ \\
\hline & 2.2. Presencia & 2,8 & - Restricciones a la presencia & $\begin{array}{l}\text { "yo tuve problemas en el colegio porque } \\
\text { cuando hacían actividades, cuando son } \\
\text { fiestas, los } 18 \text { de septiembre, eventos, actos, } \\
\text { a ella no la hacían participar y yo hablé que } \\
\text { por qué la dejaban fuera de los actos, en } \\
\text { todos los eventos y cuando eran esos actos } \\
\text { me decían mejor que no la mandara porque X } \\
\text { no iba a participar" }\end{array}$ \\
\hline & 2.3. Aprendizaje & 3,8 & $\begin{array}{l}\text { - Capacidad de aprendizaje } \\
\text { - Responsabilidad sobre el aprendizaje }\end{array}$ & $\begin{array}{l}\text { "la preocupación mayor de que él aprenda la } \\
\text { tiene el Centro de Apoyo, y con herramientas } \\
\text { adecuadas, la tutora y después la profesora" }\end{array}$ \\
\hline & 2.4. Participación & 9,9 & $\begin{array}{l}\text { - Oportunidades de participación } \\
\text { - Relación con los compañeros } \\
\text { - Información al grupo de pares } \\
\text { - Relación con los profesores }\end{array}$ & $\begin{array}{l}\text { "ella igual aprende, pero fue postergada, } \\
\text { nunca participaba, de vez en cuando } \\
\text { participaba en actividades pero, por qué } \\
\text { tengo que hablar yo para que la hagan } \\
\text { participar" }\end{array}$ \\
\hline & $\begin{array}{l}\text { 2.5. Gestión y } \\
\text { cultura } \\
\text { escolar }\end{array}$ & 0,9 & $\begin{array}{l}\text { - Actitud de la escuela } \\
\text { - Actitud del docente } \\
\text { - Condiciones del aula } \\
\text { - Coordinación de apoyos }\end{array}$ & $\begin{array}{l}\text { "ese colegio se distingue por tener una } \\
\text { integración formal y aplicada, o sea, los niños } \\
\text { saben y los padres están informados de que } \\
\text { hay niños diferentes en el colegio" }\end{array}$ \\
\hline
\end{tabular}




\begin{tabular}{|c|c|c|c|c|}
\hline Dimensiones & Categorías & $\%$ & Códigos emergentes definitivos & Citas \\
\hline \multirow{2}{*}{$\begin{array}{l}\text { 3. Apoyos para } \\
\text { la Inclusión } \\
\text { Educativa }\end{array}$} & $\begin{array}{l}\text { 3.1. Dirigidos al } \\
\text { estudiante }\end{array}$ & 6,2 & - Apoyos al estudiante & $\begin{array}{l}\text { "yo creo que era negativo, yo siempre quise } \\
\text { que los apoyos se dieran dentro de la sala } \\
\text { de clases, pero siempre me decían que el } \\
\text { decreto de integración de la X no era para } \\
\text { eso, que había otro decreto y que ese ya } \\
\text { no existía, que ese otro era para apoyar en } \\
\text { aula a los niños pero el que tenía ahora no } \\
\text { servía para eso y tenían que sacar los niños y } \\
\text { hacerles como clases aparte" }\end{array}$ \\
\hline & $\begin{array}{l}\text { 3.2. Dirigidos a la } \\
\text { familia }\end{array}$ & 5,4 & - Apoyos a la familia & $\begin{array}{l}\text { "cuando yo necesito apoyo yo sé que cuento } \\
\text { más con el Centro de Apoyos que con mi } \\
\text { familia, siento que ellos me ven más fuerte, } \\
\text { siento que ellos esperan más, siento que mi } \\
\text { familia espera tanto de mí que me da miedo } \\
\text { flaquear, entonces por eso prefiero buscar } \\
\text { más apoyo en la gente que se da cuenta } \\
\text { también que soy un ser humano común..." }\end{array}$ \\
\hline \multirow{2}{*}{$\begin{array}{l}\text { 4. Impacto de la } \\
\text { escolarización } \\
\text { sobre la familia }\end{array}$} & $\begin{array}{l}\text { 4.1. Impacto } \\
\text { en relación a la } \\
\text { madre }\end{array}$ & 12,7 & $\begin{array}{l}\text { - Bienestar de la madre } \\
\text { - Preocupaciones } \\
\text { - Expectativas } \\
\text { - Esfuerzo }\end{array}$ & $\begin{array}{l}\text { "si T está bien yo estoy bien" } \\
\text { "la inclusión ha sido difícil, uno sufre harto" }\end{array}$ \\
\hline & $\begin{array}{l}\text { 4.2. Impacto en el } \\
\text { grupo familiar }\end{array}$ & 7,0 & $\begin{array}{l}\text { - Interacción familiar } \\
\text { - Recursos económicos }\end{array}$ & $\begin{array}{l}\text { "siento que su hermana sufre algunas cosas } \\
\text { por D, ella siente algunos privilegios para él } \\
\text { y que a veces es justificado pero otras veces } \\
\text { no" }\end{array}$ \\
\hline \multirow{2}{*}{$\begin{array}{l}\text { 5. Propuestas de } \\
\text { mejoras }\end{array}$} & $\begin{array}{l}\text { 5.1. Respecto de } \\
\text { la escuela }\end{array}$ & 0.5 & - Sugerencias a la escuela & $\begin{array}{l}\text { "yo pienso que el proyecto de integración } \\
\text { no está bien enfocado, si estuviera bien } \\
\text { enfocado se lograrían más cosas, si hubieran } \\
\text { más apoyos, si fueran más constantes, } \\
\text { si fuera una profesora que empezara y } \\
\text { terminara el año pero, así como son cosas } \\
\text { del Estado, uno tiene que adaptarse a las } \\
\text { cosas del Estado" }\end{array}$ \\
\hline & $\begin{array}{l}\text { 5.2. Respecto } \\
\text { del sistema } \\
\text { educativo }\end{array}$ & 0,5 & - Sugerencias al sistema educativo & $\begin{array}{l}\text { "es necesario un cambio en los profesores } \\
\text { y no tan solo eso, tendría que haber una } \\
\text { mentalidad nueva para eso, porque si tú me } \\
\text { pides solamente algo académico para estos } \\
\text { niños no lo van a lograr, estamos hablando } \\
\text { de una integración que tiene que ser una } \\
\text { integración tanto académica como social" }\end{array}$ \\
\hline
\end{tabular}

\subsection{Dimensión 1: Conocimientos sobre Inclusión educativa}

Esta dimensión hace referencia a la primera pregunta de investigación, en relación con la perspectiva de las madres respecto de la inclusión educativa. Incluye los elementos utilizados por las participantes para definirla y su dominio sobre la normativa que la regula.
Respecto de los elementos definitorios ${ }^{3}$, ellas consideran que la inclusión educativa es un derecho $o^{4}$, tanto para el estudiante como para las madres, "es un derecho de él como persona y es

3. Las palabras resaltadas en cursiva son las categorías específicas de cada dimensión de análisis.

4. Las palabras resaltadas en negrita y cursiva son los códigos emergentes que surgieron de las entrevistas realizadas a las madres participantes. 
un derecho mío como madre el que él esté en un lugar donde se puede educar cualquiera" $\left(\mathrm{MI}^{5}\right)$. Se explicita la legitimidad que tiene participar de la educación regular de manera equitativa para todos los niños, pero también se resalta que ese derecho, actualmente, no está garantizado por el sistema educativo chileno.

Asimismo, las madres refieren algunas dimensiones que, desde su punto de vista, forman parte de lo que entienden por inclusión. Hacen alusión a la importancia de la presencia, en relación con las posibilidades o impedimentos que enfrenta el hijo para asistir a la escuela. Sin embargo, para las madres no basta solo con la presencia sino que, además, la inclusión educativa contempla bienestar y participación, lo que implica propiciar la satisfacción, motivación y participación social del hijo en la vida de la escuela, "que esté inserto pero que también sea parte, no que esté ahí sentado haciendo nada, que esté dentro del aula y participe" (MI).

Además, hablar de inclusión es hacer referencia a aprendizaje y desarrollo, poniendo énfasis en los avances evolutivos y logros en el aprendizaje alcanzados por el hijo en el contexto de la educación regular. Sin embargo, para las madres esto solo es posible cuando la inclusión educativa contempla provisión de apoyos, fundamentales para que los procesos inclusivos sean efectivos. Las madres reiteran la relevancia de asegurar la provisión de apoyos en relación con las necesidades del hijo para que el proceso se desarrolle eficazmente. Para ello, es fundamental que estos sean individualizados y pertinentes con sus necesidades y así poder continuar en la educación regular.

Respecto a la normativa que regula el acceso de alumnos con discapacidad a la escuela ordinaria, solo una de las participantes señala tener conocimiento de la misma, refiriendo que conocerla le ha permitido tomar decisiones sobre el proceso educativo del hijo. Sin embargo,

5. Todas las citas referidas por las madres han sido identificadas con la letra $\mathrm{M}$, seguida de un número que refiere la madre que ha señalado el enunciado. su valoración sobre la normativa es negativa, señalando que existe una brecha entre lo referido por la norma y lo que realmente ocurre en el sistema escolar.

\subsection{Dimensión 2: Vivencias de escolarización}

Esta dimensión aborda las preguntas de investigación referidas a la perspectiva y valoración de las experiencias vividas por las madres en torno a la escolarización de sus hijos. En su relato destacan seis aspectos fundamentales: trayectoria escolar, presencia, aprendizaje, participación, familia en la escuela, gestión y cultura escolar.

Respecto de la trayectoria escolar, todas las participantes destacan las difíciles experiencias de acceso a la educación regular. Todas coinciden en lo complejo y arduo que ha sido encontrar una escuela para sus hijos:

«Salía todos los días y todos los días llegaba llorando a la casa, entonces más que disfrutar que mi hijo iba a estar en el colegio era un pesar porque nadie me comprendía, yo decía ¿cómo nadie va a querer recibir a mi hijo si es lo más lindo?» (M6).

En general se relatan sentimientos de tristeza y frustración, con su consiguiente incidencia en el propio bienestar emocional ante la falta de oportunidades y actitudes negativas por parte de distintos tipos de establecimientos educativos. Los motivos alegados, según las madres, tienen relación con las características especiales del hijo y la falta de preparación de los docentes para enfrentar el desafío de la inclusión.

Asimismo, se muestra la necesidad de encontrar un lugar apropiado para el hijo, en el cual se concreten los mismos elementos definitorios de la inclusión educativa referidos anteriormente. Insisten que no basta con encontrar un lugar sino que este debe favorecer su bienestar emocional y asegurar la provisión de apoyos. Por otro lado, en los relatos de las madres se pone en evidencia el dilema en torno al emplazamiento educativo: educación 
regular $v /$ s educación especial. Todas las participantes coinciden en que la educación regular es la opción educativa deseada para sus hijos. No obstante, la realidad las enfrenta permanentemente a la posibilidad de tener que acceder a la educación especial ante las dificultades que se pueden vivir en la educación regular, principalmente relacionadas con el bienestar emocional del hijo, la falta de apoyos o las dificultades en el rendimiento académico. Al respecto, cabe señalar que la mayoría de las madres han decidido perseverar en la educación regular por múltiples motivaciones, entre ellas, principalmente, que a su hijo o hija le gusta asistir a su escuela.

Es importante destacar que una de las madres señala que ha optado por abandonar la educación regular y trasladar a su hija a un emplazamiento de educación especial debido a las múltiples situaciones de estrés vividas en la escuela, las cuales afectaron significativamente al bienestar emocional de la niña, "D en el mes de octubre se estresó, no quería ir al colegio, lo paso realmente mal" (M2). Resulta importante considerar que, a pesar de esta vivencia, la madre sigue manifestando su intención de regresar a corto o medio plazo a la educación regular. Las situaciones de estrés referidas estaban relacionadas con la falta de atención por parte de la escuela al bienestar emocional de su hija, tanto en el aula como en los espacios comunes, así como con la falta de provisión de apoyos para facilitar su aprendizaje y participación.

En este mismo ámbito, las madres hacen referencia a la importancia del bienestar del estudiante dentro de su comunidad educativa a la hora de hacer una valoración del proceso de inclusión educativa en el que participan. La búsqueda de bienestar emocional del hijo es el elemento que motiva, además, a mantenerse en un determinado emplazamiento o decidir gestionar un cambio de escuela, todo por acceder a mejores oportunidades educativas para el hijo.

El bienestar del estudiante es, además, un elemento esencial a la hora de hablar de calidad educativa. Las madres consideran que se puede hablar de calidad en la educación cuando se cumple con dos aspectos fundamentales para la inclusión: bienestar emocional y provisión de apoyos, "yo siento que la educación de $\mathrm{T}$ es de calidad porque T está bien” (MI), “yo creo que una educación de calidad es cuando contamos con el apoyo que ella necesita” $\left(\mathrm{M}_{4}\right)$.

Respecto de la presencia en la escuela, las madres refieren algunas restricciones a su presencia, relatando episodios en que se les solicitó no asistir a actos de celebración y a las pruebas de medición nacional de la calidad educativa. Otra restricción a la presencia es la que tiene relación con la posibilidad de que la escuela ponga término a la matrícula del hijo por dos razones: falta de adaptación a las normas escolares o por no lograr los aprendizajes mínimos esperados para su nivel escolar, evaluándose la posibilidad de derivar al hijo a una escuela especial. Esta situación es relatada por las madres como una experiencia muy angustiante.

Respecto del aprendizaje, surge entre las madres el tema de la capacidad de aprendizaje, que no está relacionado únicamente con el rendimiento académico sino con todo tipo de logros del hijo en la escuela. Mientras que algunas madres señalan la capacidad de aprender como una permanente inquietud y fuente de preocupación, para otras es una de las motivaciones para que el hijo esté en una escuela regular. Desde ambas perspectivas surge, nuevamente, la necesidad de que al hijo se le den los apoyos requeridos para facilitar este aprendizaje.

Al respecto, al preguntarles por quién ha de brindar esos apoyos y sobre en quién recae la responsabilidad sobre el aprendizaje del hijo, la mayoría de las madres focaliza esta responsabilidad en el profesional especialista o en el servicio de apoyo. Llama la atención que las familias ni siquiera esperan que la escuela asuma esta responsabilidad, tal vez por la trayectoria vivida, en la que siempre han sido otros profesionales los que han proporcionado estos apoyos.

Respecto a las oportunidades de participación que da la escuela para acceder a las distintas 
experiencias sociales y educativas que se desarrollan en la comunidad escolar, se observa una diversidad de experiencias al respecto. Hay madres que señalan que el hijo participa de todas las actividades, lo que es propiciado por la escuela, mientras que otras señalan que esa participación depende exclusivamente de ellas y de su permanente solicitud porque así sea. Según lo manifestado por las madres que explicitan restricciones a la participación, éstas se producirían por una cultura escolar poco respetuosa y comprensiva con la diversidad, que limita el acceso del alumno a experiencias escolares en donde éste requiere de mayores apoyos individuales.

En cuanto a la relación con los compañeros, la mayoría de las madres coinciden en que este aspecto es una de las razones y motivaciones para mantener el proceso de inclusión educativa, en la medida en que se trata de uno de los elementos centrales del bienestar emocional del estudiante en la escuela, "los niños siempre se encargan de acogerle cuando le ven un poco solo, los niños, los compañeritos, más que el colegio, son los compañeritos los que a mí me poner súper feliz" (M6). Sin embargo, dos madres hacen alusión a episodios aislados de abuso por parte de los compañeros.

Por otro lado, las madres opinan que es importante la información al grupo de iguales respecto de las necesidades de apoyo de su hijo, ello favorecería su aceptación por parte del grupo, así como su incorporación a todas las actividades desarrolladas en la escuela.

En cuanto a la relación con los profesores, las madres señalan que es buena, sin embargo, ninguna profundiza en su importancia para la inclusión del niño o niña.

Respecto a la relación familia-escuela, en algunos relatos se describe como estrecha y colaborativa y, en otros, que es absolutamente distante. En todos los casos esta relación está construida desde la madre hacia la escuela y directamente vinculada a la provisión de apoyos al hijo y su proyección escolar, "hemos cuidado mucho la relación con el colegio por eso mismo, hemos tratado de mantener muchas cosas en armonía con el colegio para que en el fondo nos podamos proyectar en un plazo más extenso" (MI). Las madres perciben que la relación que sostengan con la escuela es una oportunidad para generar vínculos que aseguren la permanencia del hijo en ella.

Esta misma motivación por establecer vínculos con la escuela, en beneficio de la inclusión del hijo, es la que se observa al hablar de su relación con otras familias. Todas las madres relatan que esta relación no es favorecida por las escuelas sino por ellas mismas, valorando especialmente la información que otras madres pueden dar en relación con el bienestar y participación del hijo en la escuela, o sobre los hechos cotidianos que han ocurrido en el aula (relación con los compañeros, materiales o tareas solicitadas por el profesor de aula, etc.).

Por otra parte, las madres mencionan diferentes experiencias de acceso a la información acerca del proceso educativo del hijo. Algunas señalan que no tienen dificultades en este aspecto, mientras que otras relatan que deben hacer grandes esfuerzos para acceder a la misma información. En este sentido, las madres reclaman una mayor comprensión por parte de la escuela respecto de su demanda de información, En relación con la consideración de la familia para la toma de decisiones por parte de la escuela, se observa una amplia diversidad de opiniones. Algunas madres dan cuenta de una relación colaborativa para la toma de decisiones respecto del proceso educativo del hijo. En otros casos, se refiere que las decisiones son tomadas únicamente por la escuela. Lo que sí es compartido es la importancia de ser partícipe de estas decisiones por cuanto afectan directamente al bienestar del hijo en la escuela.

En lo que respecta a la participación de la familia en la escuela, todas las madres señalan los múltiples esfuerzos que hacen por ser partícipes del proceso educativo de su hijo, con la intención de poner en evidencia su compromiso e implicación en el mismo, y favorecer su continuidad en la escuela. Se relatan 
episodios que dan cuenta del tiempo que ellas permanecen en la escuela, cómo son partícipes de las iniciativas de la comunidad de padres y madres y cómo llegan a hacerse cargo de algunas funciones escolares para permanecer cerca de sus hijos y asegurar su bienestar.

Respecto a la gestión y cultura escolar, son conscientes de la importancia que tiene para la inclusión la actitud de la escuela frente a la diversidad y la actitud del docente hacia el estudiante, sus características y necesidades de apoyo. En relación con la primera, para las madres resulta positivo que esta tenga una postura de apertura y flexibilidad frente a la diversidad dentro de su proyecto educativo institucional, así como la preocupación por el bienestar del hijo o hija.

Sobre la actitud del docente hacia el hijo, las madres coinciden en relacionar la actitud positiva del profesor con los avances en su aprendizaje. Valoran positivamente cuando observan preocupación, optimismo, atención y conocimiento de las características del hijo, así como cuando prestan atención a las necesidades de orientación e información de la familia: "la profesora de matemáticas me da harta confianza, siempre está súper dispuesta a hablar" (M6). Por el contrario, las madres destacan de forma negativa cuando el profesor no conoce las características y necesidades de apoyo de su hijo, así como cuando hay falta de atención a su bienestar y participación.

En referencia a la gestión de los apoyos, para las madres es importante que exista una coordinación entre los apoyos que se brindan al hijo para que estos sean realmente efectivos. Esta coordinación facilita que los apoyos sean pertinentes a las necesidades del hijo, individualizados y acordes a la evolución de sus necesidades.

Respecto a las condiciones del aula, las madres refieren que el número de estudiantes por aula es un elemento relevante para el desarrollo de los procesos educativos inclusivos y se reitera la idea de que un número reducido de estudiantes favorecería la individualización de los apoyos.
Asimismo, otra condición relevante señalada es la continuidad del profesor durante el año escolar, a fin de conocer el proceso educativo del hijo.

\subsection{Dimensión 3: Apoyos}

Esta dimensión responde a la tercera pregunta de investigación referida a los recursos y servicios de apoyo requeridos o brindados al estudiante y a su familia a lo largo del proceso educativo. Se organizan en dos categorías de análisis: apoyos al estudiante y apoyos a la familia.

Las madres hacen múltiples y diversas referencias a la hora de hablar de los apoyos al estudiante. La atención está puesta en la pertinencia de los apoyos, independientemente de la fuente de los mismos. Respecto a la modalidad en que se brindan los apoyos en la escuela, en algunos casos se dan dentro del aula y en otros casos fuera de ella, lo cual es valorado negativamente por las madres. Hay acuerdo en que los apoyos se realicen dentro del aula para garantizar el acceso a los aprendizajes curriculares y favorecer la participación en el aula y en la comunidad educativa.

Respecto a los apoyos a la familia, señalan que no reciben apoyo por parte de la escuela e incluso, para ellas, es esperable. Cuando es posible, estos se reciben por parte de profesionales externos a la misma. Respecto a los tipos de apoyo que necesitan, hacen referencia especialmente a dos: apoyo emocional frente a los desafíos y dificultades que se han de enfrentar a lo largo de los procesos educativos en los que participan y otros de orientación frente a inquietudes y toma de decisiones.

En relación con el primero, las madres señalan que necesitan de un soporte emocional estable en los momentos difíciles que viven junto al hijo, en términos de poder contar con una ayuda para enfrentarlos positivamente y "salir de ellos". Esta necesidad de apoyo trasciende incluso a aquellos apoyos que podrían solicitarse a su propio grupo familiar, lo que implica un desafío para cualquier profesional o servicio responsable de brindarlos. 
En relación con el segundo, las madres señalan la necesidad de contar con una orientación clara a la hora de enfrentarse a dilemas vinculados al proceso educativo del hijo, así como para comprender su evolución a lo largo del ciclo vital.

\subsection{Dimensión 4: Impacto de la escolarización sobre la familia}

Esta dimensión hace referencia a la segunda y tercera pregunta de investigación, referidas a la valoración de los procesos de escolarización y la perspectiva sobre los apoyos que reciben sus hijos y las familias durante su escolarización. Las temáticas que emergen en este ámbito son bienestar de la madre, preocupaciones, expectativas y esfuerzo.

En relación con el bienestar de la madre, es posible señalar que este se relaciona directamente con el bienestar del hijo en los distintos contextos de desarrollo. El acceso a la educación regular viene a ser un desafío tanto para el hijo como para ellas, tras el cual el bienestar de ambos puede verse afectado positiva o negativamente. Al respecto, las madres señalan que "están bien” en la medida en que el hijo "está bien”, "si T está bien yo estoy bien” (MI).

Cabe señalar que la escolarización del hijo es un proceso que las madres visualizan como una experiencia desafiante, haciendo evidente la necesidad de apoyo emocional para afrontarla de la mejor forma posible, "la inclusión ha sido difícil, uno sufre harto” $\left(\mathrm{M}_{5}\right)$.

En relación con las preocupaciones, es posible señalar que, principalmente, hay dos tipos. Las primeras se refieren a la vulnerabilidad de sus hijos a causa del TEA. Otras se centran en el proceso escolar del hijo y su proyección en la educación regular. Estas preocupaciones tienen que ver con el futuro y la incertidumbre que este les genera.

En relación con las expectativas respecto a la escuela, las madres prefieren "vivir día a día” para enfrentar mejor los desafíos que se presentan. Las expectativas de las madres están centradas, principalmente, en el bienestar emocional y no en logros académicos específicos, por lo que cabe considerar la importancia que tiene que la escuela comprenda las prioridades de la familia para con sus hijos, considerando que habitualmente el énfasis de la escuela se centra en logros de aprendizaje curricular.

En relación con el esfuerzo, las madres incluyen en sus relatos situaciones en donde destacan su tenacidad para asegurar la permanencia del hijo en la educación regular, a pesar de los múltiples desafíos o momentos difíciles a los que se han enfrentado. Las madres coinciden en que han tenido que trabajar mucho junto a sus hijos para dar continuidad a su permanencia en la escuela, a pesar del coste para su propio bienestar.

Respecto al impacto que tiene la escolarización de los hijos en la educación ordinaria sobre el grupo familiar, las temáticas que emergen en este ámbito son interacción familiar y recursos económicos.

En relación con la interacción familiar, no hay alusión explicita de las madres sobre cómo el proceso de escolarización afecta a su grupo familiar, pero sí se reitera que el proceso es generalmente vivido en soledad y con escasa participación del resto del grupo familiar, siendo ellas quienes asumen la responsabilidad de apoyar al hijo y de acompañarle en el desafío de acceder a la escuela regular. Las madres explicitan, además, que son ellas quienes toman las decisiones sobre el proceso educativo y quienes se disponen a apoyarle en la escuela. El impacto al que hacen alusión tiene que ver con la cantidad de tiempo dedicado a sus hijos con TEA en relación con el dedicado a sus hermanos, que es mucho más reducido, aspecto en torno al que muestran una gran preocupación. En referencia a los recursos económicos, las madres insisten en que estos son fundamentales a la hora de acceder a la escuela y brindar los apoyos requeridos por el hijo. Señalan que la posibilidad de pagar una escuela o no marca la diferencia en sus oportunidades educativas. Asimismo, la posibilidad de 
contar con uno u otro apoyo también está condicionada por la capacidad económica de financiarlos. Este factor viene a agudizar aun más la situación de vulnerabilidad que viven sus hijos con TEA, ya que va a depender del nivel económico de la familia el cómo y dónde se viva la experiencia de escolarización y los apoyos con que se pueda contar para hacerla efectiva.

\subsection{Dimensión 5: Propuestas de mejora}

Esta dimensión hace alusión a la quinta pregunta de investigación, que hace referencia a las propuestas que realizan las madres para avanzar hacia una educación más inclusiva. Esta dimensión contempla dos categorías de análisis: mejoras en la escuela y en el sistema educativo.

Respecto a las sugerencias dirigidas a la escuela se señala la importancia de la individualización de los apoyos, la presencia de profesionales dentro del aula, la coordinación para su provisión y el conocimiento exhaustivo de la situación de los hijos.

En cuanto a las sugerencias para la mejora de sistema educativo, se reitera la necesidad de generar un cambio de concepciones por parte de los docentes y del sistema educativo, respecto a cómo abordar la atención a la diversidad, el sentido y significado de la inclusión educativa.

Con respecto a nuestra cuarta pregunta de investigación referida a las barreras y facilitadores que identifican las madres en los procesos de escolarización vividos, ésta se responde manera transversal en las diferentes dimensiones.

\section{Discusión y Conclusiones}

Avanzar hacia una educación de calidad para todos y todas, que valore la diversidad y responda a las diferencias individuales, implica atender a lo que tienen que decir los principales involucrados en el proceso educativo, e incluir sus voces y perspectivas a la hora de pensar en las mejoras que necesita la escuela para avanzar hacia una educación cada vez más inclusiva.

El interés de nuestro trabajo ha sido abordar la inclusión educativa y la escolarización de estudiantes con TEA desde la voz de las familias, en particular de las madres, lo que nos ha permitido conocer los significados construidos por uno de los grupos fundamentales para proyectar procesos educativos inclusivos exitosos.

Centrándonos en nuestras preguntas de investigación, en relación con cuál es la perspectiva de algunas madres chilenas de estudiantes con TEA sobre el proceso de inclusión educativa, es posible concluir que este proceso es concebido como la concreción de un derecho, tanto para los hijos como para las madres, a educarse en las mismas condiciones que el resto de los niños y niñas, y a no ser excluidos y derivados a centros de educación especial.

Asimismo, las madres que han participado en el estudio entienden la inclusión educativa como un proceso que precisa la presencia del hijo en la escuela regular y que, necesariamente, debe propiciar su desarrollo, aprendizaje, bienestar y participación, en consonancia con la definición que al respecto hemos hecho sobre la inclusión educativa (Ainscow, Booth \& Dyson, 2006). Para lograrlo la escuela debe asegurar la provisión de apoyos, acordes a las necesidades de los estudiantes y a las de la familia.

Respecto al dominio de la normativa que regula la escolarización de estudiantes con discapacidad en la educación regular, la mayoría de las madres no tiene conocimiento de ella, lo que nos lleva a plantearnos de qué manera pueden hacer valer el derecho a una educación de calidad si no conocen las garantías establecidas en dicha normativa y carecen de asesoramiento en esa dirección.

En relación con la valoración de la inclusión educativa, las madres muestran un apoyo a 
la misma, consideran que puede ser positiva para el desarrollo del hijo, siendo su bienestar y participación en la escuela la motivación para continuar haciendo cualquier esfuerzo por permanecer en la educación ordinaria. No obstante, esta valoración está condicionada a la existencia de apoyos necesarios para asegurar su bienestar, aprendizaje y participación en la escuela. Lo mismo ocurre a la hora de valorar la calidad educativa; desde sus perspectivas, una educación de calidad es aquella que brinda apoyos al hijo para vivir una escolarización que propicie su desarrollo.

Cabe señalar que resulta significativo que las madres participantes, a la hora de hablar sobre inclusión educativa, utilicen los mismos elementos nucleares que aparecen en algunos trabajos de referencia sobre esta temática como son las dimensiones de presencia, aprendizaje y participación (Ainscow, Booth \& Dyson, 2006), añadiendo a estos elementos la referencia al derecho a una educación de calidad, consagrado, a su vez, en la Convención Internacional sobre los Derechos de las Personas con Discapacidad (ONU, 2006).

Respecto a nuestra segunda pregunta de investigación, sobre la valoración que hacen las madres respecto a los procesos de escolarización vividos con sus hijos, manifiestan lo difícil que fue el acceso del hijo a la escuela ordinaria, y los esfuerzos de cada una de ellas para que pueda permanecer en la misma. Esta referencia al esfuerzo que han hecho las madres es coincidente con los resultados del trabajo de Verdugo y Rodríguez (20I2), en el que también se hace alusión al agotamiento que origina la lucha por lograr que el hijo termine su proceso escolar. Las referencias al esfuerzo y a la lucha por continuar en la escuela ordinaria son coincidentes, además, con el estudio de Rogers (2007), en el que se recoge la experiencia de padres de niños con discapacidad en la educación regular, con el estudio de O'Connor (2007), centrado en el análisis de las preocupaciones de los padres sobre la inclusión educativa o con el de Kluth et al. (2007), sobre historias de familias que buscan mejores alternativas de escolarización para sus hijos con discapacidad.
Este esfuerzo indudablemente tiene implicaciones en el bienestar emocional de la madre y del grupo familiar, y la percepción de bienestar de la familia se ve condicionada por el del hijo, en la misma línea de los resultados obtenidos en otros trabajos sobre calidad de vida en familias con hijos con discapacidad (González del Yerro et al., 20I3; Poston et al., 2004). La interrelación existente entre las dimensiones de calidad de vida individual y las de calidad de vida familiar supone que, muchas de las necesidades de la familia queden supeditadas a la búsqueda del bienestar de uno de sus miembros, la cual generalmente posterga sus propias necesidades por satisfacer las necesidades del más vulnerable (Poston et al., 2004). Esta situación tiene implicaciones importantes a la hora de considerar los apoyos que se brindan a los alumnos con discapacidad y a sus familias, por lo que la escuela debe asumir la incidencia que tiene su acción en la calidad de vida de ambos.

Por otro lado, volviendo a la valoración del proceso de escolarización, las madres destacan la falta de oportunidades para que el hijo acceda a la escuela, vinculadas a su discapacidad, existiendo pocas alternativas educativas dispuestas a permitir su presencia en la escuela ordinaria. Las dificultades para acceder y permanecer en ella tienen relación con el desfase curricular que presenta el hijo en los aprendizajes esperados para cada nivel educativo, situación que se incrementa a medida que crece. Esta situación es concordante con lo obtenido en otros trabajos como los de Echeita et al. (2009), Kluth et al. (2007) o Verdugo y Rodríguez (20I2), en donde las familias participantes de su estudio hacen referencia a que esto se agudiza al llegar a la educación secundaria.

Respecto a nuestra tercera pregunta de investigación, sobre cuál es la perspectiva sobre los apoyos recibidos por sus hijos y sus familias durante los procesos de escolarización en los que participan, la mayoría hace una valoración negativa de los apoyos brindados por la escuela. Entre las dificultades manifestadas cabe destacar la modalidad en la que son brindados, 
habitualmente fuera del aula, la baja frecuencia de los mismos, así como la falta de coordinación entre los profesionales que los proporcionan. Además, las madres manifiestan no tener expectativas de que esta situación mejore.

En relación a cómo deberían ser los apoyos para sus hijos, según las madres, estos deben ser individualizados y pertinentes con sus necesidades, con el contexto y las diferentes etapas de su desarrollo. Las madres prefieren que los apoyos educativos se lleven a cabo dentro del aula, a fin de ajustar los contenidos y la enseñanza a la realidad del grupo/curso. Este requerimiento es coherente con la forma en que se entienden los apoyos en un paradigma inclusivo (Gallego, 20 I I; Sandoval, Simón y Echeita, 20I2). Asimismo, se espera una gestión coordinada y articulada a la hora de brindarlos, tanto entre los profesionales que los proporcionen como en la coordinación entre estos y la familia. En este sentido, cabe señalar que la demanda de las madres por una gestión coordinada de los mismos es común a otras investigaciones desarrolladas con familias cuyos hijos requieren de una atención específica por parte de diversos profesionales (Martin et al., 2009) y es un aspecto contemplado en las propuestas de intervención con familias (Turnbull, Turnbull y Kyzar, 2009).

Por otro lado, las madres esperan que se contemplen apoyos personalizados que puedan favorecer el acceso de los hijos a los aprendizajes curriculares. Asimismo, hacen referencia a la necesidad de que estos contemplen tanto su desarrollo académico como social y emocional en la escuela. Consideran que deben ir dirigidos no solo al logro de determinadas competencias curriculares instrumentales, sino también al logro de habilidades cognitivas como afectivas.

En este contexto, el docente se enfrenta entonces a un importante desafío a la hora de atender a una amplia gama de necesidades de apoyo en el aula, para lo que es necesario poner en marcha estrategias de programación de la enseñanza que permitan responder a las necesidades de todos los alumnos, coherente con las propuestas de un Diseño Universal para el Aprendizaje (DUA) (Cast, 2OII).
En cuanto a los apoyos a la familia, las madres manifiestan la necesidad de contar con apoyo emocional ante los desafíos y dificultades, así como de orientación frente a inquietudes y toma de decisiones, lo que implica establecer relaciones positivas y colaborativas entre las familias y los profesionales (Moliner, 2008; Simón, 20I3; Turnbull, Turnbull y Kyzar, 2009), a fin proveer apoyos de manera eficaz, pertinentes con las necesidades del hijo y la familia (Poston et al., 2004; Turnbull, 2003).

En relación con nuestra cuarta pregunta de investigación, referida a qué barreras identifican las madres en el proceso de escolarización de sus hijos, es posible señalar que delimitaron varias condiciones que se traducen en limitaciones para los procesos de escolarización en la educación ordinaria. Se identifican de esta forma barreras a la presencia, percibiendo una falta de apertura y preparación por parte de las escuelas para posibilitar el acceso de estudiantes con discapacidad a la educación regular y la falta de proyectos educativos comprensivos con la diversidad, condiciones coincidentes con los hallazgos de O'Connor (2007) y Rogers (2007).

Asimismo, se señala la falta de oportunidades de inclusión educativa, considerando que en Chile todavía se propicia el acceso a centros ordinarios solo a aquellos estudiantes con discapacidad que presentan mínimos requerimientos de apoyos, lo cual deja a muchos de ellos excluidos de la educación ordinaria.

Como otra barrera a la escolarización, y en la línea de lo obtenido en otros estudios, las madres convergen en señalar la falta de individualización de la enseñanza para el estudiante con discapacidad que accede a la educación regular (Kluth et al., 2007; O’Connor, 2007; Rogers, 2007; Verdugo, Sainz y Rodríguez, 2009). En conexión con este aspecto, se apunta la falta de preparación de los docentes para atender a las distintas necesidades educativas presentes en el aula, como ha sido expresado también en los trabajos de O'Connor (2007), Rogers (2007), Verdugo, Sainz y Rodríguez (2009). Las madres reconocen la importancia de enriquecer la 
formación docente en el ámbito de la atención a la diversidad, sin dejarla únicamente en manos de especialistas y profesionales de apoyo, por cuanto eso ha favorecido una falta de implicación por parte de los docentes de aula a la hora de responsabilizarse del aprendizaje de todos sus estudiantes, sobre todo de los que requieren de apoyos específicos, atribuyendo esa responsabilidad solo a los "especialistas" (Sandoval, Simón y Echeita, 20 I2) y no a la escuela, la que debería responder a la diversidad de todo su alumnado.

Respecto al bienestar y participación, las madres señalan que la escuela centra toda su atención en el rendimiento académico, sin atender los ámbitos de desarrollo personal y social, con una tendencia a responsabilizar al estudiante de sus propias dificultades de aprendizaje. Asimismo, se señala una falta de consideración de la familia por parte de la escuela durante los procesos de escolarización y toma de decisiones, hallazgo coincidente con el trabajo de O'Connor (2008). Del mismo modo, en la línea de lo señalado por Kluth et al. (2007), se desataca la falta de apoyos adecuados para el estudiante y su familia por parte de la escuela. También se insiste en la falta de coordinación entre los profesionales a la hora de proporcionar los apoyos, así como de información sobre el proceso escolar, al igual que lo observado en las familias de los trabajos de Frederickson et al. (2004), Verdugo, Sainz y Rodríguez (2009) e Yssel et al. (2007).

Respecto a los facilitadores de los procesos de escolarización delimitados por las madres, se puede señalar que, desde su perspectiva, estos surgen en la medida en que el entorno escolar genera estrategias para erradicar o mitigar las barreras que hemos descrito anteriormente. En relación con nuestra última pregunta de investigación, sobre qué propuestas realizan las madres para avanzar hacia una educación más inclusiva, las sugerencias que hacen a la escuela y al sistema educativo tienen relación directa con las necesidades del hijo y con la experiencia particular de cada una de ellas a lo largo del proceso de escolarización.

A modo de síntesis podemos identificar, desde la perspectiva de las madres participantes, factores críticos de la inclusión educativa y del proceso de escolarización de sus hijos. En la Tabla 3 se presentan estos factores, que son interdependientes.

Finalmente, los resultados del estudio permiten poner de manifiesto cómo la valoración que

Tabla 3. Factores críticos de la inclusión educativa y del proceso de escolarización de estudiantes con discapacidad

\begin{tabular}{|c|c|}
\hline Ámbito & Factores críticos \\
\hline En relación con el estudiante & $\begin{array}{l}\text { - Bienestar emocional } \\
\text { - Participación en la escuela }\end{array}$ \\
\hline En relación con la familia & $\begin{array}{l}\text { - } \text { Bienestar de la familia } \\
\text { necesidades del estudiante } \\
\text { necursos económicos de la familia para responder a las }\end{array}$ \\
\hline En relación con la escuela & $\begin{array}{l}\text { - Oportunidades de acceso a la escuela ordinaria } \\
\text { - Individualización de los programas educativos } \\
\text { - La actitud del docente frente a la diversidad } \\
\text { - } \text { Proyecttura de la escuela frente a la diversidad } \\
\text { - Posibilidad de participación de la familia en el proceso } \\
\text { - } \text { educativo } \\
\text { - Relaciones entre la familia y los profesionales }\end{array}$ \\
\hline En relación con los apoyos & $\begin{array}{l}\text { - Pertinencia de los apoyos al hijo } \\
\text { - Pertinencia de los apoyos a la familia } \\
\text { - Coordinación de los apoyos }\end{array}$ \\
\hline
\end{tabular}


hacen las madres participantes sobre los procesos de escolarización de sus hijos está directamente relacionada con el nivel de satisfacción que tengan con los apoyos que brindan las escuelas para asegurar su presencia, aprendizaje y participación. Asimismo, cabe destacar la importancia de escuchar las voces de las familias a la hora de proyectar mejoras a los procesos educativos inclusivos para estudiantes con discapacidad, así como la necesidad de implementar procesos de evaluación sistemática en los que las familias sean el motor fundamental para avanzar hacia una educación efectivamente más inclusiva.

Respecto a las proyecciones del estudio, consideramos pertinente profundizar en las diferentes variables de las familias (sociales, personales, demográficas, etc.) y de los centros educativos (relacionadas con culturas, políticas y prácticas) que podrían influir en los procesos inclusivos y en la percepción que tienen las familias de los mismos, con el fin de avanzar hacia el logro de de una educación comprensiva con la diversidad.

\section{Limitaciones del estudio}

En cuanto a las limitaciones de esta investigación, además de las cautelas en la generalización de los resultados debido a la muestra, queremos destacar el hecho de que se trata únicamente de las opiniones y perspectivas de las madres. Sería importante conocer y enriquecer esta visión con las voces de los padres y de los propios alumnos, así como con las de otros miembros del sistema familiar, sin olvidar en este proceso la incidencia de factores de los propios centros escolares. 
Ainscow, M.; Booth, T. \& Dyson, A. (2006): Improving schools, developing inclusion, London: Routledge.

Ainscow, M. \& West, M. (eds.) (2006): Improving urban schools: Leadership and collaboration, Maidenhead: Open University Press.

Ainscow, M. et al. (2012): "Making schools effective for all: rethinking the task", School Leadership \& Management, 32(3): 197-213.

American Psychiatric Association (s.f.): Diagnostic and Statistical Manual of Mental Disorders (DSM) (en línea). http://www.psychiatry.org/ practice/dsm.

Braun, V. \& Clarke, V. (2006): "Using thematic analysis in psychology", Qualitative Research in Psychology, 3(2): 77-IOI.

CAST (20I I): Universal Design for Learning. Guidelines version 2.o., Wakefield: MA Author (en línea). http://www.udlcenter.org/aboutudl/ udlguidelines', acceso el 5 de enero de 2014.

Dobbins, M. \& Abbott, L. (20I0): “Developing partnership with parents in special schools: parental perspectives from Northern Ireland", Journal of Research in Special Educational Needs, Io(I): 23-30.

Echeita, G. y Simón, C. (2007): “La contribución de la educación escolar a la calidad de vida de las personas con discapacidad. Ante el desafío de su inclusión social”. En: De Lorenzo, R. y Cayo Pérez, L.: Tratado sobre Discapacidad, Madrid: Thomson \& Aranzadi: I I03-II34.

Echeita, G. et al. (2009): "Paradojas y dilemas en el proceso de inclusión educativa en España", Revista de Educación, 349: I 53-I 78 (en línea). http://www.revistaeducacion.mec.es/re349/ re349_08.pdf.

Echeita, G. et al. (2008): La inclusión educativa del alumnado con necesidades educativas especiales, asociadas a discapacidad, en España. Un estudio prospectivo y retrospectivo de la cuestión, vista desde la perspectiva de las organizaciones no gubernamentales de personas con discapacidad, Informe de investigación no publicado.

Frederickson, N. et al. (2004): "Mainstream-special inclusion partnerships: pupil, parent and teacher perspectives", Internacional Journal of Inclusive Education, 8(I): 37-57.

Gallego, C. (20II): "El apoyo inclusivo desde la perspectiva comunitaria", Revista Interuniversitaria de Formación del Profesorado, 25(I): 93-I09.

Giné, C. (2004): "Servicios y Calidad de Vida para las Personas con Discapacidad Intelectual”, Revista Española sobre Discapacidad Intelectual, 35(2): I-I4.

González del Yerro, A. et al. (2013): "La calidad de vida de las familias de personas con discapacidad intelectual. Un estudio cualitativo realizado en la Comunidad de Madrid”, Revista Española de Orientación y Psicopedagogía, 24(I): 93-I09.

Jackson, B.; Renwick, R. \& Fudge, A. (2008): "Parental Perspective of the Quality of Life in School Environments for Children with Asperger Syndrome", Focus on Autism and Other Developmental Disabilities, $23(4)$ : 242-252.

Kluth, P. et al. (2007): "Going Away to School: Stories of Families Who Move to Seek Inclusive Educational Experiences for their Children with Disabilities", Journal of Disability Policy Studies, I 8(I): 43-56.

Leal, L. (2008): Un enfoque de la discapacidad intelectual centrado en la familia, Madrid: FEAPS.

Martin, E. et al. (2009): La respuesta educativa al alumnado con trastornos de conducta, Madrid: El Defensor del Menor de la Comunidad de Madrid.

Mertens, D.M. (2010): Research and evaluation in education and psychology: integrating diversity with quantitative and qualitative, and mixed methods, Londres: SAGE Publications. 
MINEDUC (2005): Política Nacional de Educación Especial "Nuestro compromiso con la diversidad”, Santiago de Chile: Ministerio de Educación, Gobierno de Chile.

Moliner, O. (2008): “Condiciones, procesos y circunstancias que permiten avanzar hacia la inclusión educativa: retomando las aportaciones de la experiencia canadiense", Revista Electrónica Iberoamericana sobre Calidad, Eficacia y Cambio en Educación, 6(2): 27-44.

O’Connor, U. (2007): “Parental views on inclusion: the Northern Ireland perspective", International Journal of Inclusive Education, II (5-6): 535-550.

ONU (2006): Convención internacional sobre los derechos de las personas con discapacidad (en línea). ‘http://www.un.org/spanish/disabilities/ convention/qanda.html, acceso el 7 de enero de 2014 .

ONU (1989): Convención de Naciones Unidas sobre los derechos del niño (en línea). http:// www.ohchr.org/SP/ProfessionalInterest/Pages/ CRC.aspx', acceso el 6 de enero de 2014.

Parsons, S.; Lewis, A. \& Ellins, J. (2009): “The views and experiences of parents of children with autistic spectrum disorder about educational provision: comparisons with parents of children with other disabilities from an online survey", European Journal of Special Needs Education, 24(I): 37-58.

Poston, D. et al. (2004): "Calidad de vida familiar: un estudio cualitativo”, Siglo Cero, 35(3), 2II: 3 I -48 .

Rodrigo, M.J. et al. (2008): Preservación Familiar. Un enfoque positivo para la intervención con familias, Madrid: Pirámide.

Rodrigo, M.J. y Palacios, J. (1998): Familia y Desarrollo Humano, Madrid: Editorial Alianza.

Rogers, C. (2007): “Experiencing an 'inclusive' education: parents and their children with 'special educational needs'”, British Journal Sociology of Education, 28(I): 55-68.

Salend, S. \& Garrick, L. (2002): "What do families have to say about inclusion?", Teaching Exceptional Children, 35(I): 62-66.
Sandoval, M.; Simón, C. y Echeita, G. (20I2): "Análisis y valoración crítica de las funciones del profesorado de apoyo desde la educación inclusiva", Revista de Educación, núm. extraordinario: I I7-I 37 .

Schippers, A. \& Van Boheemen, M. (2009): "Family quality of life empowered by familyoriented support", Journal of Policy and Practice in Intellectual Disabilities, 6(I): 19-24.

Simón, C. y Echeita, G. (20I0): "Educación inclusiva y participación de las familias: dilemas, paradojas y esperanzas". En: Echeita, G. el al. (coords.): La inclusión en la educación democrática. El éxito para todos y todas a lo largo de la vida, Madrid: Proyecto Atlántida: I08-ı 8.

Turnbull, A.P. (2003): "La calidad de vida de la familia como resultado de los servicios: el nuevo paradigma”, Siglo Cero, 34(207): 59-73.

Turnbull, A.P.; Turnbull, H.R. y Kyzar, K. (2009): "Cooperación entre familias y profesionales como fuerza catalizadora para una óptima inclusión: enfoque de los Estados Unidos de América", Revista de Educación, 349: 69-99.

UNESCO (2004): Temario Abierto sobre Educación Inclusiva: Materiales de Apoyo para Responsables de Políticas Educativas, Santiago de Chile: Oficina Regional de Educación de la UNESCO para América Latina y el Caribe (OREALC).

UNESCO (1994): La Declaración de Salamanca el Marco de Acción para las Necesidades Educativas Especiales, Madrid: UNESCO.

Verdugo, M.A. (2009): "El cambio educativo desde una perspectiva de calidad de vida", Revista de Educación, 349: 23-43.

Verdugo, M.A. y Rodríguez, A. (20I2): “La inclusión educativa en España desde la perspectiva de alumnos con discapacidad intelectual, de familias y de profesionales”, Revista de Educación, 358: 450-470.

Verdugo, M.A.; Sainz, F. y Rodríguez, A. (2009): Escala de calidad de vida familiar, Salamanca: Universidad de Salamanca, INICO.

Yssel, N.et al. (2007): "Views of Inclusion. A comparative study of parents' perceptions in South Africa and the United States", Remedial and Special Education, 28(6): 356-365. 